\title{
Optical Properties of Type-I and II Quantum Dots
}

\author{
F. Iikawa ${ }^{1}$, M. P. F. Godoy ${ }^{1}$, M. K. K. Nakaema ${ }^{1}$, M. J. S. P. Brasil ${ }^{1}$, M. Z. Maialle ${ }^{2}$, \\ M. A. Degani ${ }^{3}$, E. Ribeiro ${ }^{4}$, G. Medeiros-Ribeiro ${ }^{4}$, W. Carvalho Jr. ${ }^{4}$, and J. A. Brum ${ }^{1,4}$ \\ ${ }^{1}$ Instituto de Física, Universidade Estadual de Campinas, Campinas, SP, Brazil \\ ${ }^{2}$ Liceu Vivere, Pirassununga, SP, Brazil \\ ${ }^{3}$ Haras Degani, Itatiba, SP, Brazil \\ ${ }^{4}$ Laboratório Nacional de Luz Sincrotron, Campinas, SP, Brazil
}

Received on 30 March, 2003

\begin{abstract}
This paper focuses on recent results on the optical properties of self-assembled quantum dots involving typeI InGaAs/GaAs and type-II InP/GaAs interfaces. In the first part, we focus on the InGaAs/GaAs quantum dots, that were used to study the influence of a two-dimensional electron gas on the optical emission of single quantum dots. In the second part, we present the results on type-II InP/GaAs quantum dots. In this system, we observed an experimental evidence indicating that the model used to interpret the blue shift of the type-II quantum well optical emission cannot be applied to type-II quantum dots.
\end{abstract}

\section{Introduction}

Among nano-structured semiconductor systems, the development of the self-assembled quantum dots (QDs) has allowed the investigation of the quantum properties of zerodimensional systems [1]. The band alignment of the interfaces in self-assembled QDs is one of the key parameters that determines both electron and hole spatial distribution inside or around the dots. From the point of view of optical properties this charge distribution results in completely distinct behaviour for the QD emission. In type-I QDs, photocreated electrons and holes are both confined in the QD layer; for type-II structures, either the electron or the hole is confined in the dot and the other carrier remains in the barrier material, but surrounding the QD due to the Coulomb attraction between the e-h pair. In this scenery the magnitude of the electron-hole Coulomb interaction is strongly dependent on the spatial charge distribution. Here we present a review of optical studies in two systems with interfaces type-I [2] and II [3] that explore the particularities of the band alignment on the QD optical properties.

Due to the small lattice mismatch, $\mathrm{In}_{x} \mathrm{Ga}_{1-x} \mathrm{As} / \mathrm{GaAs}$ self-assembled QDs for low $\mathrm{x}$ are formed on top of a wide wetting layer quantum well (WL) compared to InAs/GaAs QDs. The resulting coupling between their quantum states makes it possible to investigate the interaction between carriers in a quasi zero-dimensional state and carriers in a quasi-two dimensional state. The first part of the paper shows the results of a systematic study using microphotoluminescence spectroscopy $(\mu$-PL) that clearly indicates the influence of the 2DEG in the WL on the QD optical emission lines of InGaAs/GaAs self-assembled QDs.

In the second part we present the results of optical measurements in type-II self-assembled InP/GaAs QDs. In this system electrons are confined in the InP dots and holes are localized at the GaAs layer around the dots. The optical properties of type-II QDs, such as GaSb/GaAs [4-7], Ge/Si [8] and recently InP/GaAs [3,9], are less investigated compared to type-I systems. The spatial separation of electrons and holes at distinct material layers leads to a spatial indirect recombination resulting in relatively longer radiation lifetimes, as compared to type-I systems, which is a very attractive characteristic for device applications such as optical memories [10]. Previous works with various type-II QDs systems have always reported a large blue shift of their broad emission band for increasing excitation intensities, which has been often considered as a main characteristic of typeII quantum dots and wells $[4,5,9]$. This blue shift is usually attributed to an increasing quantum confinement of the carriers around the dots in the triangular-like potential built at the barrier interface for an increasing photocreated carrier density. Alternatively, some authors consider that the blue shift may be dominated by other effects such as state filling and many particle effects $[6,7,11]$. Nevertheless, a detailed understanding of these contributions has not been established yet. Here we discuss $\mu$-PL results that rule out the hypothesis of the blue shift observed for type-II QDs being due to the band bending of the interface potential. The occupation of the higher energy states is therefore a more likely mechanism responsible for this effect.

\section{InGaAs/GaAs quantum dots}

The samples were grown by molecular beam epitaxy on a GaAs (001) substrate. The self-assembled QDs are formed on the top of an $\operatorname{In}_{x} \mathrm{Ga}_{1-x}$ As WL (nominal thickness of 58 monolayers and composition of $\mathrm{x}=0.24)$. The QDs are covered by a $30 \AA$ GaAs layer followed by a $1400 \AA$ $\mathrm{Al}_{0.3} \mathrm{Ga}_{0.7} \mathrm{As}$ barrier and a $100 \AA \mathrm{GaAs}$ cap layer. The 
2DEG is provided by a Si delta doping separated by $300 \AA$ from the $\mathrm{AlGaAs} / \mathrm{GaAs}$ heterojunction. The carrier density at the WL is $\sim 3 \times 10^{11} \mathrm{~cm}^{-2}$ and the average dot concentration was estimated to be of the order of $1 \times 10^{9} \mathrm{~cm}^{-2}$ [12].

The $\mu \mathrm{PL}$ experiments were performed using a coldfinger He-cryostat (sample temperature $\sim 6 \mathrm{~K}$ ), the 4880 $\AA$ Aline of an Ar-ion laser, a 1/2 m single spectrometer with a spectral resolution of $\sim 0.4 \mathrm{meV}$ and a S1 photomultiplier. The laser beam was focused on the sample by a $50 \mathrm{X}$ objective with a spot diameter of $\sim 4 \mu \mathrm{m}$. A thin Al-foil mask with micrometer pinholes was used to identify a fixed focusing position at the sample surface. The 2DEG was controlled either by applying a DC-gate voltage $\left(V_{g}\right)$ between the sample surface and the 2DEG or by varying the excitation intensity $\left(P_{\text {Laser }}\right)$.

Figure 1 shows typical $\mu \mathrm{PL}$ spectra of InGaAs/GaAs QDs. The strong emission line is due to the recombination in the WL and for lower energies we observed several sharp peaks due to the emission from individual QDs, which are shown in the inset of Fig. 1. The WL emission was used as a sensor of the 2D electron gas density in the WL. The change of $V_{g}$ results in changing of 2D electron density which affects directly the WL optical emission due to the variation of the built-in electric field. The red shift of WL emission peak as the 2D electron density increases observed in our sample (not shown here) is an indication of the increase of the built-in electric field in the WL quantum well, known as the Stark shift effect.

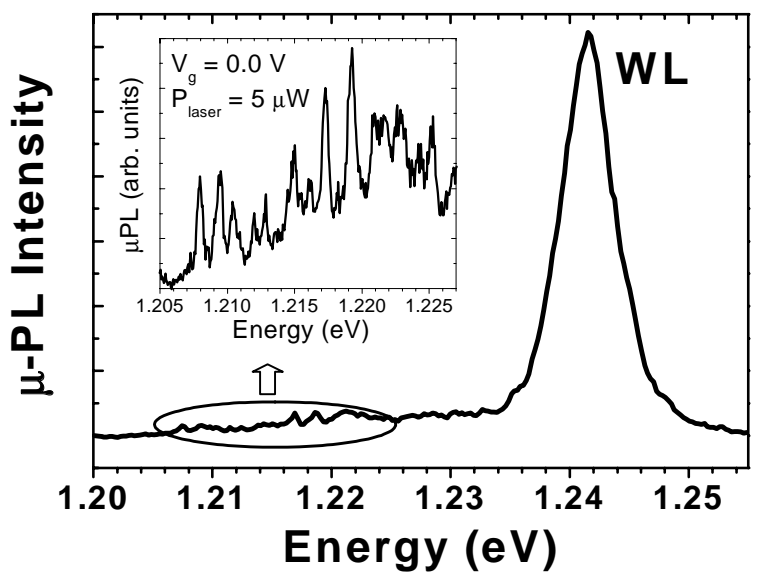

Figure 1. $\mu$-PL spectra of InGaAs/GaAs QDs. The inset shows the QD emission region in more detail.

Since the height of the QDs is smaller than the WL thickness, we expect that the potential of the QDs is very shallow relative to the WL quantum well resulting in only a single discrete level for the electrons in the QD [12]. Since the QD is the lowest energy state of our structure, the build up of a 2DEG in the WL indicates that the QD conduction band levels are completely filled.

Figure 2 shows the behavior of the QDs emission lines as a function of $V_{g}$ maintaining a constant $P_{\text {Laser }}=5 \mu \mathrm{W}$. As $V_{g}$ increases, the peaks $\mathrm{A}$ and $\mathrm{B}$, for instance, show a blue shift, $\sim 0.6 \mathrm{meV}$, in contrast to the expected red shift of the WL. We also observed a slight broadening (up to $\sim$
$0.8 \mathrm{meV}$ ) and an intensity decrease. At $V_{g}>1.60 \mathrm{~V}$ all emission peaks have almost vanished. Both the energy position and the line width show a strong non-linear behavior as a function of $V_{g}$, varying significantly for $V_{g}>1.0 \mathrm{~V}$. We did not observe any variation either in the WL or in the QD emission lines for $V_{g}<0$. The 2DEG density should decrease for negative $V_{g}$ down to a certain limit (the 2D gas itself is one of the conductor plates of our capacitor).

A complete picture for explaining those observations depends on complex calculations considering the electronic structure and the presence of the electron gas. We may discuss however some fundamental points that strongly contributes to those effects. The modulation-doped heterojunction creates a non-constant built-in electric field in the QW and QD regions that has a non-linear dependence on the 2DEG density and $V_{g}$. The InAs self-assembled QDs are expected to be lens-shaped, thus presenting an asymmetry along the growth direction. Figure 3 shows the results of the calculation, using split operator method, of electron-hole transition energy as function of the field $\mathrm{F}$, which simulates the built-in field of the sample. We considered a graded In composition variation in the dot along the growth direction based on the results reported in the literature $[13,14]$. The field range expected in our case is indicated in the Fig. 3 by a rectangle. We see that the WL transition energy presents a red shift, while it is obtained a blue shift for the QD emission, as also observed in previous works $[14,15]$.

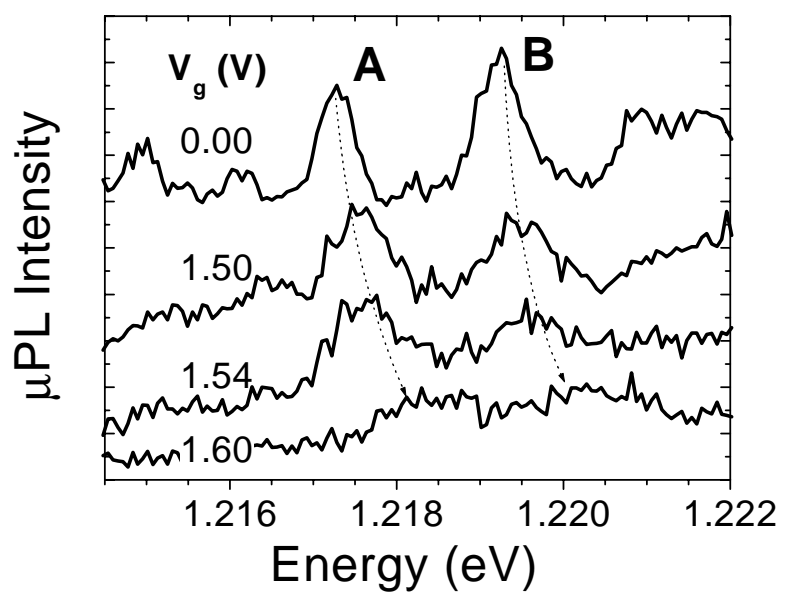

Figure 2. Typical $\mu$-PL of InGaAs/GaAs QDs.

The increase of positive $V_{g}$ implies in an increase of the electron gas density and a variation of the self-consistent potential of the structure. The increase of the electron gas density may contribute to the observed broadening since the recombination in a QD induces excitations of the electron sea in the WL. As the 2DEG density in the WL increases the Fermi energy increases and energy range of the occupied continuous 2D states that allow electron-electron scattering also increases, which should result in an increase of the QD emission line width. An alternative origin for the broadening of the QD emission lines is the tunneling of holes out of the dots towards the GaAs buffer layer. However, we do not 
expect a variation of this effect with $\mathrm{Vg}$, since $V_{g}$ is applied between the WL and the sample surface such that the resulting variation of the potential barrier for holes is rather small. Concerning the quenching of the QD emission lines for increasing 2DEG densities, it may have its origin in a weaker capture efficiency of holes by the QDs as the built-in electric field in the QD increases. In order to clarify the origin of those results we are now calculating the electronic states considering the interaction between electrons in the QD and WL.

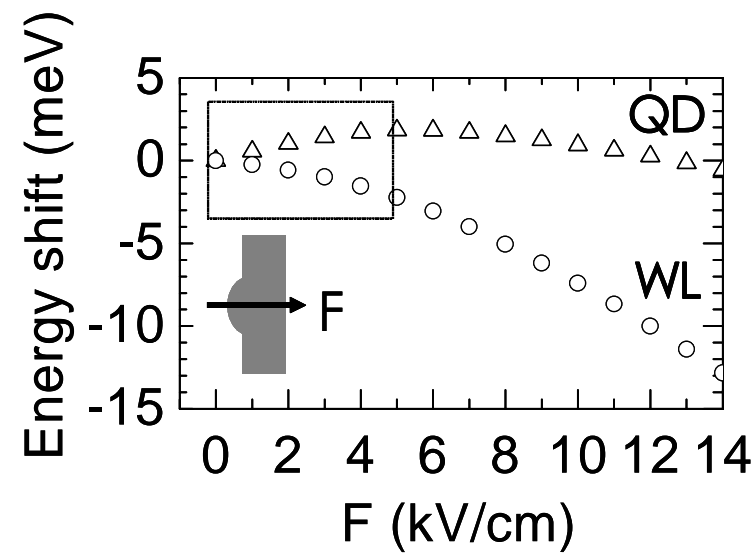

Figure 3. Results of the calculation of the energy shift of QDs in the presence of the electric field $\mathrm{F}$.

\section{InP/GaAs type-II quantum dots}

The structures were grown by low pressure Metal Organic Chemical Vapor Deposition (MOCVD). Initially a $300 \mathrm{~nm}$ $\mathrm{GaAs}$ buffer layer was grown at $640{ }^{\circ} \mathrm{C}$ after which the GaAs substrate temperature was lowered to $550{ }^{\circ} \mathrm{C}$ without growth interruption. An InP layer 2.3-2.4 monolayer thick was then grown at $1.5 \mathrm{ML} / \mathrm{s}$ producing self-assembled quantum dots. A GaAs $50 \mathrm{~nm}$ capping layer was grown followed by a control InP layer under the same conditions as the buried InP layer. A previous atomic force microscope (AFM) analysis shows the formation of InP dots with a large size dispersion with an average height of $4.5 \pm 0.5 \mathrm{~nm}$ and diameters of $30 \pm 3 \mathrm{~nm}$. The surface density of dots is estimated to be of the order of $2 \times 10^{10} \mathrm{~cm}^{-2}$.

Micro-photoluminescence measurements were performed using the same setup described above. Based on the dot density obtained by AFM measurements we thus estimate that our system probes an ensemble of $\sim 2500$ dots. We also used time integrated PL (TIPL) using a Ti-Sa pulsed laser (pulse width of $2 \mathrm{ps}$ ).

Figure 4 shows the excitation intensity dependence of the PL and TIPL spectra. We observe in both figures a broad band emission (line width $\sim 70 \mathrm{meV}$ ) attributed to the QDs luminescence that exhibits a clear blue shift as the excitation intensity increases. The spectra of TIPL for the high excitation regime show two peaks, which we attributed to QDs (low energy) and WL (high energy) optical emissions.
Based on TIPL results we fitted two Gaussian line shape to all spectra of Fig. 4. We obtained the blue shift of the peak position for both emission bands. The blue shifts are 25 $\mathrm{meV}$ for the QD emission band and $40 \mathrm{meV}$ for the WL. The behaviour of the WL emission band is predicted for type-II quantum wells; it is due to the variation of the interface triangle-like potential, which leads to an increase of the transition energy as the carrier density increases. For QDs this interpretation is no longer valid. The experimental results discussed in the follow show the contradiction with this model.
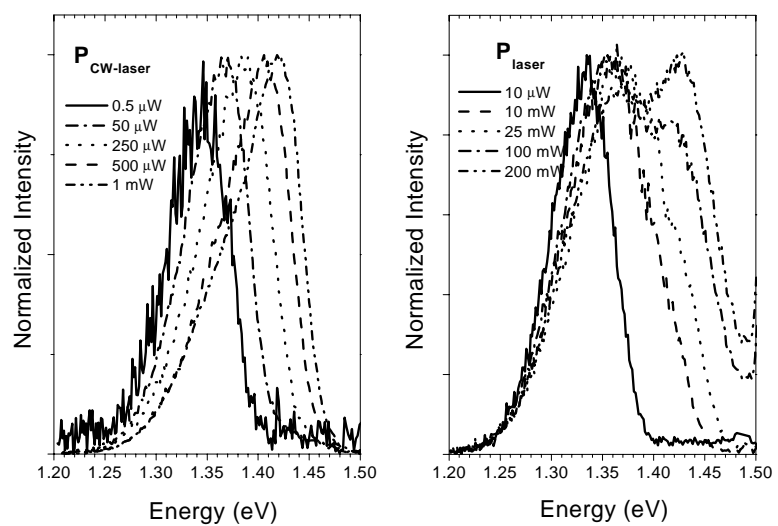

Figure 4. PL (left) and TIPL (right) spectra of InP/GaAs QDs for several laser powers.

Figure 5 shows the results of $\mu \mathrm{PL}$ measurements corresponding to a small energy range of the broad QD emission band. On top of the broad band background, the $\mu \mathrm{PL}$ spectra present several reproducible sharp features with line widths of the order of $1 \mathrm{meV}$ or narrower. Figure 5 shows two different scans measured under the same experimental conditions (curves $\mathbf{a}$ and $\mathbf{b}$ ) from the same area exhibiting a very good reproducibility of all spectral features. We also present an additional scan under the same experimental conditions but focusing the laser spot at a distinct area of the sample (curve c), which clearly presents a different set of features. The sharp features can be associated to statistical fluctuations of the emission from the ensemble of $\sim 2500 \mathrm{InP}$ dots probed by the laser beam. These fine structures is reproduced by simulated spectra for an ensemble of dots emitting sharp lines of equal amplitude randomly distributed within a broad Gaussian distribution. We have performed simulations and showed that for the line width of our broad band emission the sharp features are observable for ensembles as large as 10.000 dots. Even though the observed features do not correspond to the emission of individual dots, we can infer the behaviour of a single dot emission from the observation of those features, which is corroborated by their reproducibility.

$\mu$ PL spectra obtained for various excitation intensities at a given area of the sample are also shown in Fig. 5. We observed that, in contrast to the results from Fig. 4, the sharp features does not shift in energy as the excitation intensity is varied within the same range as used in Fig. 5. This result 
demonstrates that the energy position for the PL of a single QD is not affected by the increase of the carrier density.

The ground state of our type-II QDs is given by an electron confined at the dot and a hole at the GaAs layer bound to the electron via Coulomb interaction, which is a OD state similar to a hole bound to a negative acceptor-impurity. In order to produce a continuous energy shift of this 0D state it is necessary to vary the occupation of extended states with a continuum spectrum that interact with the 0D state. The extended states in our system are those where the electrons are at the wetting layer and the holes at the barrier adjacent to the wetting layer interfaces. The main effect of the occupation of these states on the QD emission lines is to screen out the Coulomb interaction between the carriers forming the OD state. Therefore, for the power intensity range used in our measurements we expect that the screening effect of the QD states by the extended states should be negligible and no continuous energy shift of the QD emission lines should occur.

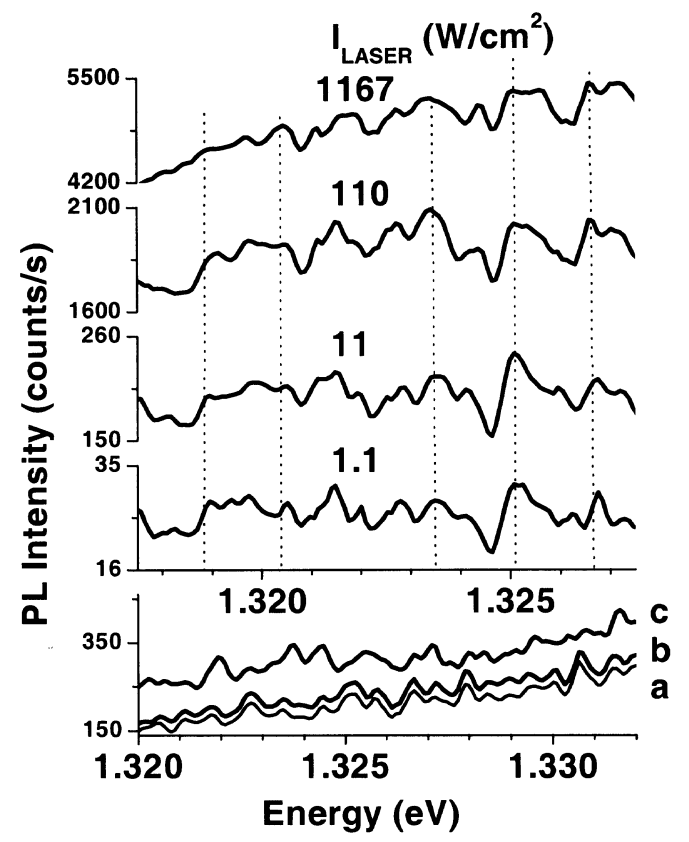

Figure 5. Optical emission of ensemble of QDs for different excitation intensity. The labels $\mathbf{a}, \mathbf{b}$ and $\mathbf{c}$ are described in the text.

An additional point that must be considered is that the increase of the excitation intensity should also result in an increase of the occupation of the QDs. However, 0D states are completely discrete. The addition of an extra carrier in the dot causes a major discrete change in its effective potential, and consequently in the dot emission energies, due to the Coulomb interaction between the carriers. Different charge configurations of the QD result in optical emissions with distinct energies that may occur at different times but cannot coexist. Therefore, we expect that an increase of the excitation intensity will essentially result in an increase of the probability that the QD be occupied by more than two carriers, and this in turn will be reflected by an increase of intensity of the emission lines corresponding to those QD charge configurations.
Therefore, the large blue shift of the QD broad emission band can only be attributed to the occupation of higher energy states, including smaller dots and larger dots with more than two carriers. Concerning the case of QDs with more than two carriers, it is important to consider that the spatial separation of carriers on type-II QDs weakens the electronhole interaction as compared to type-I QDs. This effect disturbs the balance among the electron-electron, hole-hole and electron-hole interactions and leads to a significant increase of the energy necessary to add a second carrier/exciton into the QD [11]. In the case of the biexciton and the charged exciton with the extra carrier being the one confined at the dot, it was shown that their binding energies for a type-II QD are enhanced by a factor as large as 16 relative to a type-I QD [11]. Therefore the increasing probability of occupation of the QDs excited states with increasing excitation intensities may also have a significant contribution to the asymmetry of the broad emission band observed in Fig. 4.

In summary, we have presented experimental evidence that the strong blue shift observed in type-II QDs is not due to a carrier density dependent triangular potential at the dot interface. The evidence is based on the invariance of the sharp features observed by micro-photoluminescence measurements as a function of the excitation intensity. We attribute the observed blue shift of the broad emission band to the filling of higher energy states.

\section{Acknowledgements}

The authors acknowledge the Brazilian agencies: FAPESP, CNPq and FINEP for financial support.

\section{References}

[1] P. M. Petroff, A. Lorke, and A. Imamoglu, Phys. Today, May, $46(2001)$

[2] M. K.K. Nakaema, M.J.S.P. Brasil, F. Iikawa, E. Ribeiro, T. Heinzel, K. Ensslin, G. Medeiros-Ribeiro, P.M. Petroff, and J.A. Brum, Physica E, 12, 872 (2002)

[3] M. K. K. Nakaema, F. Iikawa, M. J. S. P. Brasil, E. Ribeiro, G. Medeiros-Ribeiro, W. Carvalho Jr., M. Z. Maialle, and M. H. Degani, Appl. Phys. Lett. 81, 2743(2002)

[4] F. Hatami, N. N. Ledentsov, M. Grudmann, J. Böhrer, F. Heinrichsdorff, M. Beer, D. Bimberg, S. S. Ruvimov, P. Werner, U. Gösele, J. Heydenreich, U. Richter, S. V. Ivanov, B. Ya. Meltser, P. S. Kop'ev, and Zh. I. Alferov, Appl. Phys. Lett. 67, 656 (1995).

[5] C.-K. Sun, G. Wang, J. E. Bowers, B. Brar, H.-R. Blank, H. Kroemer, and M. H. Pilkuhn, Appl. Phys. Lett. 68, 1543 (1996).

[6] L. Mueller-Kirsch, R. Heitz, A. Schliwa, O. Stier, D. Bimberg, H. Kirmse, and W. Neumann, Appl. Phys. Lett. 78, 1418 (2001).

[7] R. A. Hogg, K. Suzuki, K. Tachibana, L. Finger, K. Hirakawa, and Y. Arakawa, Appl. Phys. Lett. 72, 2856 (1998).

[8] P. Schittenhelm, M. Gail, J. Brunner, J. F. Nützel, and G. Abstreiter, Appl. Phys. Lett. 67, 1292 (1995). 
[9] B. Wang and S.-J. Chua, Appl. Phys. Lett. 78, 628 (2001).

[10] M. C. Bödefeld, R. J. Warburton, K. Kanai, J. P. Kotthaus, G. Medeiros-Ribeiro, and P. M. Petroff, Appl. Phys. Lett. 74, 1839 (1999).

[11] Ph. Lelong, K. Suzuki, G. Bastard, H. Sakaki, and Y. Arakawa, Physica E 7, 93 (2000).

[12] E. Ribeiro, F. Cerdeira, M.J.S.P. Brasil, T. Heinzel, K. Ensslin, G. Medeiros-Ribeiro, P.M. Petroff, J. Appl. Phys. 87 7994 (2000).
[13] I. Kegel, T. H. Metzger, A. Lorke, J. Peisl, J. Stangl, G. Bauer, J. M. Garcia, and P.M. Petroff, Phys. Rev. Lett., 85, 1694 (2000).

[14] P.W. Fry, I.E. Itskevich, D.J. Mowbray, M.S. Skolnick, J.J. Finley, J.A. Barker, E.P. O'Reilly, L.R. Wilson, I.A. Larkin, P.A. Maksym, M. Hopkinson, M. Al-Khafaji, J.P.R. David, A.G. Cullis, G.Hill, J.C. Clark, Phys. Rev. Lett. 84733 (2000)

[15] S. Raymond, J.P. Reynolds, J.L. Merz, S. Fafard, Y. Feng, and S. Charbonneau, Phys. Rev. B58, R13415, 1999 